\title{
Genomic sequence of infectious bursal disease virus from Zambia suggests evidence for genome re- assortment in nature
}

\author{
Authors: \\ Christopher J. Kasanga ${ }^{1}$ \\ T. Yamaguchi ${ }^{2}$ \\ H.M. Munang'andu ${ }^{3}$ \\ P.N. Wambura ${ }^{1}$ \\ K. Ohya ${ }^{4}$ \\ H. Fukushi ${ }^{4}$ \\ Affiliations: \\ ${ }^{1}$ Department of Veterinary \\ Microbiology and \\ Parasitology, Sokoine \\ University of Agriculture, \\ Tanzania \\ ${ }^{2}$ Faculty of Agriculture, \\ Tottori University, Japan \\ ${ }^{3}$ Department of Paraclinical \\ Sciences, University of \\ Zambia, Zambia
}

${ }^{4}$ Faculty of Applied Biological Sciences, Gifu University,

Japan

Correspondence to:

Christopher Kasanga

Email:

chrisskasa@gmail.com

Postal address:

PO Box 3019, Chuo kikuu,

Morogoro, Tanzania

How to cite this abstract: Kasanga, C.J., Yamaguchi, T., Munang'andu, H.M., Wambura, P.N., Ohya, K. \& Fukushi, H., 2012, 'Genomic sequence of infectious

bursal disease virus from

Zambia suggests evidence for genome re-assortment in nature', Onderstepoort Journal of Veterinary Research 79(2), Art. \#473, 1 page. http://dx.doi. org/10.4102/ojvr.v79i2.473

Note:

Proceedings of the

Conference of the Southern

African Centre for Infectious Disease Surveillance 'One Health' held at the National Institute for Communicable Diseases, Johannesburg, July 2011.

(C) 2012. The Authors. Licensee: AOSIS OpenJournals. This work is licensed under the Creative Commons Attribution License.
Infectious bursal disease virus (IBDV) is a bi-segmented RNA virus, which belongs to the genus Avibirnavirus of the family Birnaviridae. Two serotypes, 1 and 2, exist in IBDV. The serotype 1 IBDV s are the causative agents of infectious bursal disease (IBD) in chickens worldwide and lead to immunosuppression in young birds. Genome re-assortment has been speculated to occur and contribute to the emergence of new IBDV strains. However, evidence was lacking until recently when two re-assortant viruses were detected in China. In this study, we determined the complete nucleotide sequence of an IBDV, designated KZC-104, from a confirmed natural IBD outbreak in Lusaka, Zambia in 2004. The genome consisted of 3074 and 2651 nucleotides in the coding regions of segments $\mathrm{A}$ and $\mathrm{B}$, respectively. Alignment of both nucleotide and deduced amino acid sequences, and phylogenetic analysis revealed that the genome segment A of KZC-104 was derived from a very virulent strain, whereas its segment $B$ was derived from a classical attenuated strain. On BLAST search, the full-length segments A and B sequences showed $98 \%$ closest nucleotide homology to the very virulent strain D6948 and $99.8 \%$ closest nucleotide homology to the classical attenuated strain D78, respectively. This is a unique IBDV reassortant strain, which has emerged in nature involving segment $B$ of a live attenuated vaccine. This observation provides direct evidence for the involvement of vaccine strains in the emergence of reassortant IBDV in the field. Taken together, these findings suggest an additional risk of using live IBDV vaccines, which may act as genetic donors for genome re-assortment. Further studies are required to investigate the epidemiology and biological characteristics of reassortant strains so that the appropriate and safe IBDV vaccines can be recommended. 